\title{
Worldwide fluctuations in dengue fever cases related to climate variability
}

\author{
Marianne J. Hopp ${ }^{1,3, *}$, Jonathan A. Foley ${ }^{2}$ \\ ${ }^{1}$ International Research Institute for Climate Prediction (IRI), Lamont-Doherty Earth Observatory (LDEO), \\ Columbia University, 61 Route 9W, Palisades, New York 10964, USA \\ ${ }^{2}$ Center for Sustainability and the Global Environment (SAGE), Gaylord Nelson Institute for Environmental Studies, \\ University of Wisconsin-Madison, 1710 University Avenue, Madison, Wisconsin 53726, USA
}

${ }^{3}$ Present address: 540 Foxview Place, Ottawa, Ontario, K1K 4C4, Canada

\begin{abstract}
Dengue fever is the most significant mosquito-borne viral disease of humans and is a leading cause of childhood deaths and hospitalizations in many countries. Variations in environmental conditions, especially climatic parameters, affect the dengue viruses and their principal mosquito vector, Aedes aegypti, but few studies have attempted to quantify these relationships at the global scale. Here we use a numerical model to simulate the response of Ae. aegypti to observed climatic variations from 1958 to 1995 and to examine how modelled Ae. aegypti populations may be related to dengue and DHF cases worldwide. We find that variations in climate can induce large variations in modelled Ae. aegypti populations at the global scale. Furthermore, these climate-induced variations in modelled Ae. aegypti populations are strongly correlated to reported historical dengue/DHF cases, especially in Central America and Southeast Asia. These results suggest that potential dengue caseloads could be anticipated using seasonal climate forecasts to drive the mosquito model, thus providing a useful tool in public health management.
\end{abstract}

KEY WORDS: Dengue $\cdot$ Climate $\cdot$ Aedes aegypti $\cdot$ Mosquito $\cdot$ Model $\cdot$ Population dynamics $\cdot$ Climate forecasts

\section{INTRODUCTION}

Dengue fever potentially affects 2.5 billion people in more than 100 tropical and sub-tropical countries and is considered the most important vector-borne viral disease in the world (WHO 1999, Rigau-Pérez et al. 1998). Current estimates suggest that up to 50 million dengue cases occur annually, including 500000 cases of the more serious related illness, dengue haemorrhagic fever (DHF) (Gubler 1998, Pinheiro \& Chuit 1998, WHO 2000, 2001). The social and economic costs of this disease are comparable to those of malaria, tuberculosis and hepatitis. We have developed a global-scale, climate-driven model of the principal dengue mosquito Aedes aegypti (Hopp \& Foley 2001).
In this study we use this numerical mosquito model to simulate the response of Ae. aegypti to observed climatic variations from 1958 to 1995, and examine how the modelled Ae. aegypti populations relate to reported dengue and DHF cases worldwide. We find that a strong correlation exists between the modelled mosquito densities and reported dengue/DHF cases in regions such as Southeast Asia and Central America. Such relationships may be utilized to develop early warning systems of potential dengue outbreaks by using seasonal climate forecasts to drive the mosquito model.

Classical dengue fever, also known as breakbone fever, is distinguished by headache, a sudden onset of fever, sore muscles and joints, with occasional nau- 
sea/vomiting and rash; these symptoms may last for several days (WHO 1998). Dengue haemorrhagic fever $(\mathrm{DHF})$, is a more serious illness mainly affecting children and young adults; symptoms include a sudden onset of fever and haemorrhagic manifestations that result in significant fluid loss and may lead to shock (WHO 1998). Approximately $5 \%$ of DHF cases are fatal (Gubler \& Clark 1995). A prior infection with one of the 4 dengue viruses results in a greater probability of contracting DHF (WHO 2000). As no vaccine presently exists, the only method of controlling or preventing dengue and DHF is to combat the mosquito vectors (WHO 1998). Several species of Aedes mosquitoes carry the dengue viruses (e.g. Aedes albopictus, Ae. polynesiensis); however, the primary vector for dengue is Ae. aegypti, a peridomestic (living in and around human dwellings), day-biting mosquito, which feeds preferentially on human blood (Gubler 1997, Rohdain \& Rosen 1997).

Environmental conditions strongly control the geographic distribution and abundance of Aedes aegypti (Christophers 1960, Focks et al. 1993a,b, Rueda et al. 1990). Breeding habitats for the mosquito consist of any type of water-holding container, from tree holes or leaves to man-made cisterns, discarded bottles and tires. These man-made habitats are abundant near urban populations, where the food supply (human blood) for gravid (pregnant) female mosquitoes is also plentiful. In these environments, climatic variables such as temperature, humidity, and precipitation significantly influence mosquito development and survivorship. Temperature affects the rate of development in the different mosquito life stages, as well as dengue viral development. Mosquito survival rates are temperature dependent; the presence of water is necessary for egg laying and hatching and for larval survivorship, and relative humidity affects adult mosquito mortality (Christophers 1960, Watts et al. 1987, Rueda et al. 1990, Focks et al. 1993a,b, Hopp \& Foley 2001).

Many factors have a significant effect on the distribution of dengue, dengue/DHF caseloads and on the magnitude of an epidemic, including socioeconomic variables such as the presence of mosquito monitoring and control programs, and the use of window screens and air conditioning (Gubler 1998, Reiter 1998, 2001, Reiter et al. 2003). Dengue/DHF caseloads also depend on which of the 4 dengue viruses are circulating in an area. Exposure to one of the viruses provides a lifelong immunity to that virus, but it increases the probability of contracting DHF upon exposure to one of the other 3 dengue viruses (WHO 2000).

The presence and abundance of Aedes aegypti is vital to the transmission of the disease. Therefore, we examined the relationship between the modelled mosquito densities and reported dengue caseloads.

\section{METHODS}

\subsection{Global-scale mosquito model}

Previous studies have examined the effect of climate on dengue transmission based on global warming scenarios (Jetten \& Focks 1997, Martens et al. 1997, Patz et al. 1998). These models use some temperaturedependent calculations, including adult mosquito survivorship and dengue viral development, in addition to temperature-independent epidemiological factors such as the ability of an infected mosquito to infect a human and vice-versa, and the likelihood that an infected person will recover (Martens et al. 1997). Our modelling effort differs in that we focus specifically on the population dynamics of the principal dengue vector, Aedes aegypti.

To examine the global-scale relationships between climate, Aedes aegypti populations, and cases of dengue/DHF, we used a numerical model of mosquito population dynamics. The model is driven by precipitation, temperature, relative humidity and solar radiation (input) to describe the effects of global-scale climatic conditions on Ae. aegypti abundances (output). The model is based on the CIMSiM mosquito model (Focks et al. 1993a,b), which was originally designed to examine Ae. aegypti populations in specific cities. In this study, we simplified the model for application at the global scale as described in Hopp \& Foley 2001. The model simulates the relationship between climate and the development, population dynamics, and potential distribution of Ae. aegypti. It uses a daily timestep and operates on a $1^{\circ} \times 1^{\circ}$ latitude-longitude grid ( $100 \mathrm{~km}$ on a side). By tracking the abundance, age, and development of the mosquito in its 4 life stages (egg, larval, pupal and adult), the model simulates a life table of 200 cohorts.

The model uses an enzyme kinetics algorithm in which temperature-dependent enzyme reactions determine the development rate for each cohort in its particular life stage (Sharpe \& DeMichele 1977). Mosquito development accumulates each day, based on the mean daily air temperature, and it is complete when it reaches a specified level. In order for larvae to pupate, a minimum weight must be reached. Larvae weight is calculated using a differential equation that incorporates the effect of temperature. The number of offspring an adult female produces is a function of her larval weight. As a simplification in modelling at the global scale, we assume food availability is not a limiting factor (Hopp \& Foley 2001).

Aedes aegypti survival rates are temperature and moisture dependent. Temperature dependent survival rates are calculated using daily minimum and maximum temperatures. Studies indicate eggs can survive 
desiccation for many months, but water $(>10 \mathrm{~mm})$ is required in the ovipositional (egg laying) container for a mature egg to hatch. Larvae require water for survival, but pupae do not, and vapor pressure deficit, based on relative humidity, affects adult mortality (Christophers 1960).

To account for water availability for oviposition, egg hatching and larval survivorship, the model uses a hypothetical container in each grid cell, with a given depth of $20 \mathrm{~cm}$ and an area of $1 \mathrm{~m}^{2}$. The number of mosquitoes in each grid cell is calculated per $\mathrm{m}^{2}$ of container surface water. The $20 \mathrm{~cm}$ depth is an average depth for typical containers used for oviposition, e.g. discarded bottles, tires and water storage drums. By excluding human population densities and socioeconomic factors that might determine the types and densities of appropriate oviposition containers, and by using only one type of container per grid cell, we strictly assess the effects of climate on mosquitoes. This allows us to compare modelled mosquito densities between years and locations without having to account for regional differences and changes in containers over time.

The model calculates the availability of water (in the container) for mosquitoes on a daily basis, using a simple treatment of the surface water balance. Differences between precipitation and evaporation drive changes in water depth. Additional precipitation above the container's maximum depth runs off from the container. A simple energy balance approach simulates evaporation rates (Prentice et al. 1993). To assess the effects of climate on mosquitoes (as opposed to human activity) we do not account for manual filling of water storage containers. People living in dry conditions will often store water in containers near their homes, providing breeding grounds for mosquitoes, sometimes resulting in dengue epidemics during or following droughts (Shope 1991, Gagnon et al. 2001).

To drive the model, we used the CRU05 historical climate dataset. This $0.5^{\circ} \times 0.5^{\circ}$ latitude-longitude resolution ( $50 \mathrm{~km}$ on a side) dataset consists of observed monthly climate data from 1901 to 1995 assembled by the University of East Anglia's Climatic Research Unit (New et al. 1999, 2000). We interpolated the CRU05 data to a $1.0^{\circ}$ resolution and ran the model from 1958 to 1995 using precipitation, temperature (including min. and max. temperatures), relative humidity and fractional cloud cover. The monthly data were linearly interpolated to produce quasi-daily values.

\subsection{Dengue fever case reports}

To evaluate how climate-induced variations in Aedes aegypti population dynamics relate to changes in dengue fever cases, we compiled reports of dengue and DHF cases. For the Americas, we used data from 1980 to 1997 supplied to the Communicable Diseases Program of the Pan American Health Organization (PAHO), a regional office of the World Health Organization (WHO) (PAHO 1994, 1997). In instances where both dengue and DHF data were supplied for a country, we used the combined total. For the rest of the world we used GIDEON (Global Infectious Disease \& EpidemiOlogy Network), a software program containing caseload and diagnostic data on over 300 diseases for more than 200 countries, gathered from WHO statistics, journals and periodicals, and national health ministries (Berger 1995).

To improve the statistical robustness, only countries with at least 5 years of dengue/DHF caseload data were included. Several countries were excluded from analysis due to poor data quality; e.g. some countries, particularly in Africa, contained case data that were not reported for specific years. Also, the relatively coarse $1^{\circ} \times 1^{\circ}$ latitude-longitude resolution grid of our model excluded the use of dengue data from several islands and small nations, e.g. in the western Pacific and Caribbean.

The reliability of the dengue and DHF case data is an important factor, as dengue fever is often misclassified or not reported (Hales et al. 1999). In this study we have used all the available dengue and DHF data, but an option in future studies may be to use DHF case data exclusively; DHF is more likely to be properly diagnosed and reported, because the symptoms are more severe and people are more likely to seek medical treatment. The source of the dengue/DHF data is another significant factor. For the Americas, we calculated the correlation coefficients using both the PAHO and GIDEON case data. As these data sets have different sources, they often differ both in number of years of data, and in the actual number of cases reported where years do overlap (Tables 1 \& 2). Nevertheless, we explored whether significant correlations between the modelled mosquito densities and case data exist using all available dengue and DHF data.

Some countries may have experienced increases in DHF cases due to the introduction of additional dengue viruses. If only 1 of the 4 dengue viruses is circulating in a country, there will be few, if any, DHF cases. Exposure to additional dengue viruses, however, results in a greater probability of acquiring DHF (WHO 2000).

To determine the relationship between the simulated variations in mosquito potential abundance and the variations in the reported number of dengue/DHF cases, we compared the annual case data with the modelled mosquitoes, by country for 1958-1995. Our study included PAHO and GIDEON data for 20 countries in the Americas and 12 countries in Asia and the western Pacific. 

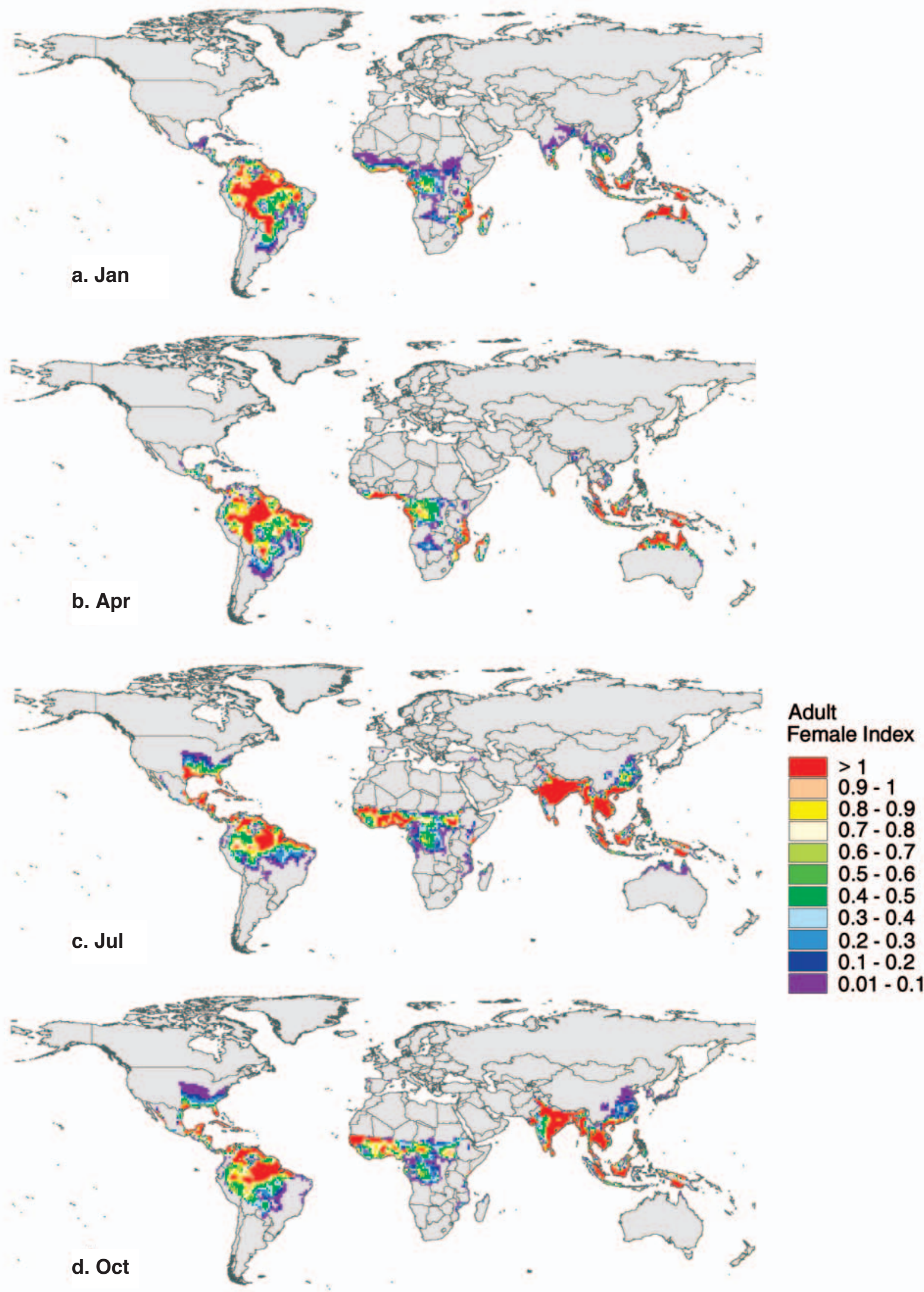

Fig. 1. Modelled potential adult female mosquito Aedes aegypti index (mosquitos $\mathrm{m}^{-2}$ of container surface water), for (a) January, (b) April, (c) July, (d) October 
To account for changes in the number of dengue/ DHF cases between 1958 and 1995 due to non-climate factors such as population increases or migration, we detrended the case data and the modelled mosquito data, and calculated the correlation coefficients between detrended and non-detrended values.

\section{RESULTS}

\subsection{Model}

Seasonal variation in the density (mosquitoes $\mathrm{m}^{-2}$ of container surface water) and distribution of the modelled adult female mosquito population is shown in Fig. 1a-d for January, April, July and October, respectively, averaged over 1961-1990. The modelled mosquito is strongly influenced by temperature, as evidenced by the northward and southward seasonal shifts in distribution. Precipitation is also an important factor for the mosquito's survival. The onset of the Asian monsoon around the middle of June provides ample precipitation for mosquito survival, and this is reflected by the dramatic increase in mosquito density from April to July in India and Southeast Asia.

To determine the response of the mosquito model to its climatic inputs, or to determine the climatic sensitivity of the model, we correlated month-to-month variations in simulated larvae densities against variations in the individual climatic parameters that drive the model (Fig. 2). The strongest relationship occurs between variations in mosquito larvae densities and temperature (Fig. 2a). The next strongest correlations occur with precipitation (Fig. 2b) and relative humidity (Fig. 2c), followed by fractional cloud cover (Fig. 2d).

The most significant connections between modelled mosquito and temperature variations occur in the moist tropical regions. This is expected given that temperature affects mosquito development rates, oviposition,
Correlation coefficient for larvae vs. temp

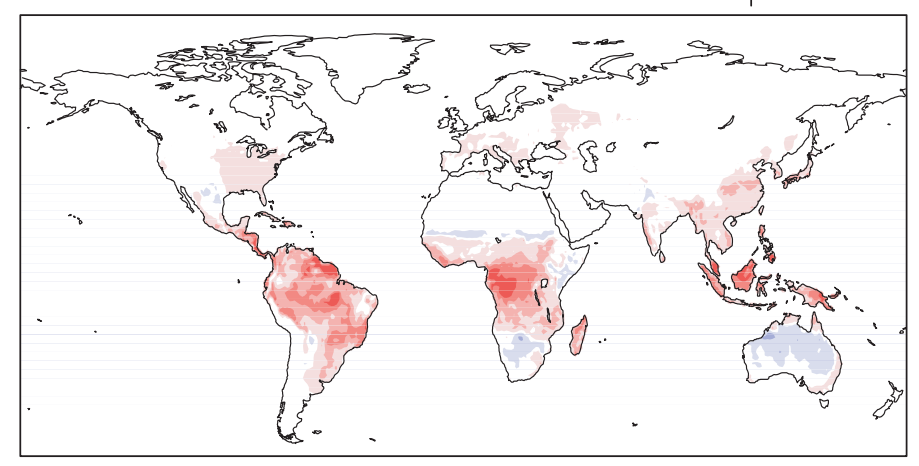

Correlation coefficient for larvae vs. precip

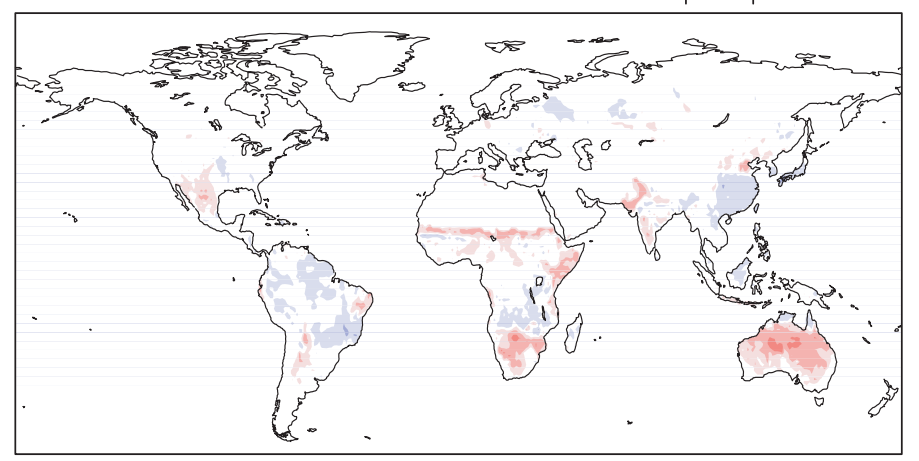

Correlation coefficient for larvae vs. rh

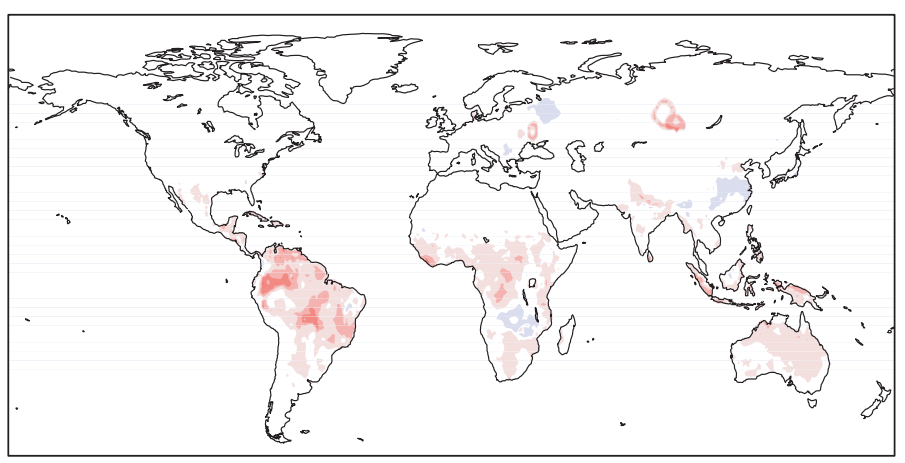

Correlation coefficient for larvae vs. cld

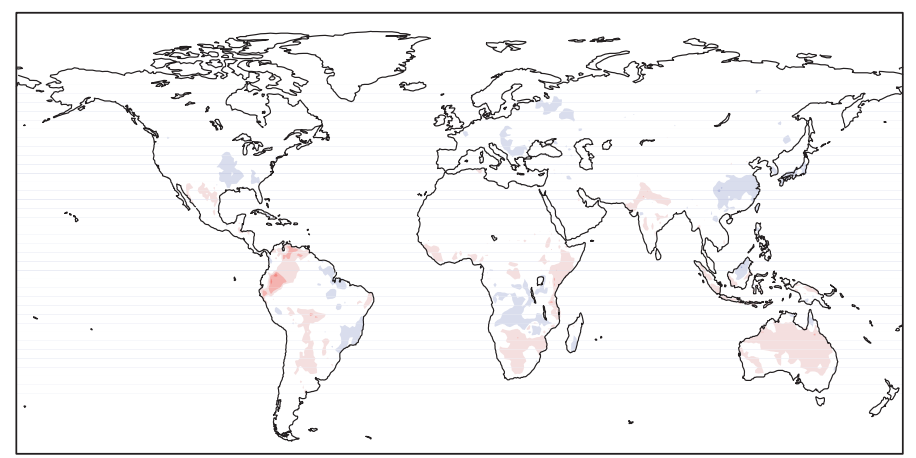

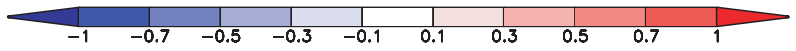

Fig. 2. Linear correlation coefficients between modelled mosquito (Aedes aegypti) larvae and CRU05 climate data, based on monthly anomalies (deviations from the long-term mean), for 1958 to 1995. Correlation coefficients between larval anomalies and (a) temperature anomalies, (b) precipitation anomalies, (c) relative humidity anomalies, (d) cloud cover anomalies 


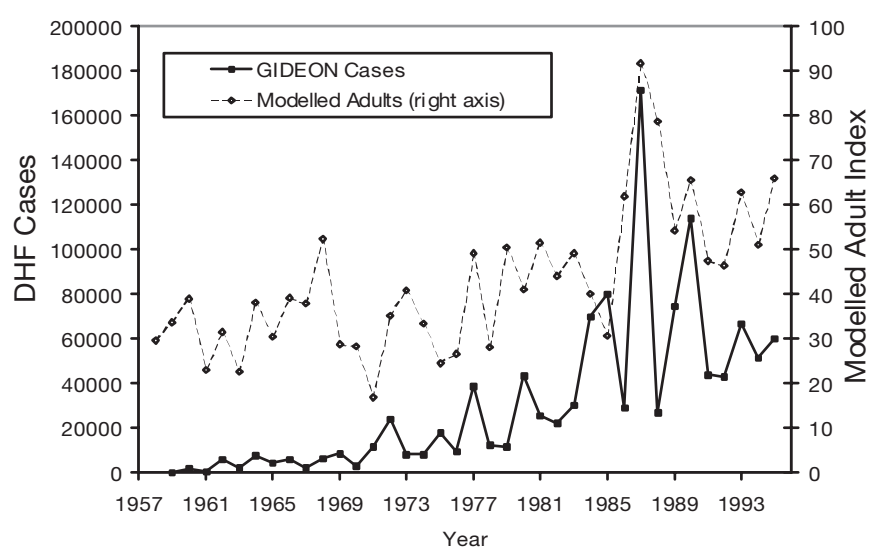

Fig. 3. Thailand: DHF cases (GIDEON dataset) and modelled adult mosquito (Aedes aegypti) index. Correlation coefficient $=0.68^{*}\left(0.43^{*}\right.$ detrended $) ;{ }^{*} \mathrm{p}<0.05$

and survivorship in the model. As these moist areas receive sufficient rainfall for larval survival and oviposition, correlations with relative humidity (important for adult survival) are stronger than with precipitation or fractional cloud cover (which is also used in the model to calculate water availability).

Drier regions, such as northeastern Brazil, and parts of Australia and Africa (e.g. Sahel, Horn of Africa, and southern Africa), exhibit stronger correlations with precipitation than with temperature, relative humidity or cloud cover. In these areas, moisture is the limiting factor for mosquito survivorship and oviposition in the model.

\subsection{Comparison with dengue case data}

Dengue outbreaks require the presence of dengue viruses in addition to the mosquitoes. However, for the purposes of this study we have only examined the dengue case data and the modelled mosquito densities, i.e. ignoring the possible presence or absence of the virus itself.

The detrended dengue/DHF case data and modelled mosquito densities of 3 of the 12 Southeast Asian countries have statistically significant $(\mathrm{p}<0.05)$ positive correlations: Thailand (Fig. 3), Indonesia (Fig. 4), and Vietnam, all reporting DHF cases (Table 1). The yearto-year fluctuations in modelled mosquito densities for these countries correspond well with the peaks in the reported dengue/DHF cases (Figs. 3 \& 4).

The results obtained with non-detrended (raw) data indicate that the majority of the countries ( 7 out of 12) in Southeast Asia and the western Pacific have statistically significant $(p<0.05)$ positive correlations between modelled mosquito densities and number of reported dengue cases (Table 1). Of these 7 countries,

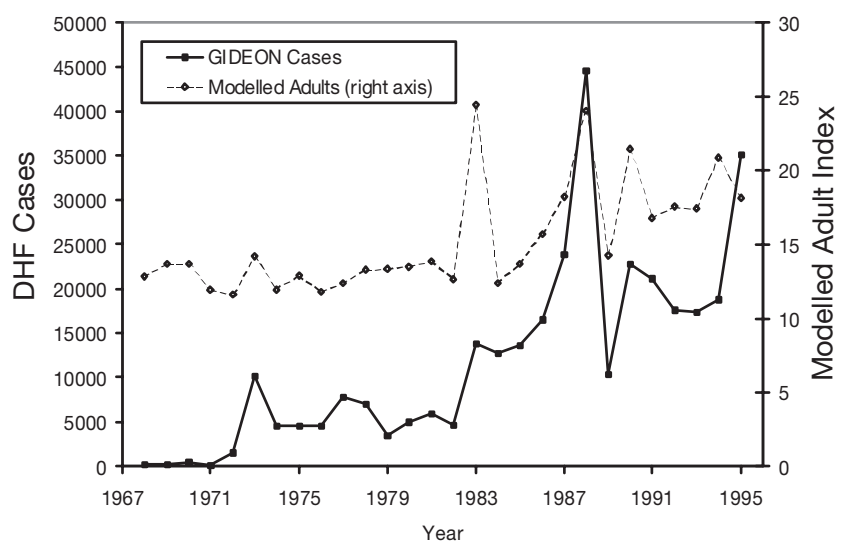

Fig. 4. Indonesia: DHF cases (GIDEON dataset) and modelled adult mosquito (Aedes aegypti) index. Correlation coefficient $=0.77^{*}\left(0.58^{*} \text { detrended }\right)_{i}{ }^{*} \mathrm{p}<0.05$

Cambodia, Indonesia, Laos, Philippines, Thailand and Vietnam reported only DHF data, while Malaysia reported dengue and DHF cases (see Table 1). The number of reported years of case data for these countries averaged about 24 .

In the Americas, with the PAHO dataset, there were statistically significant $(p<0.05)$ correlations between the non-detrended modelled mosquito densities and dengue/DHF cases for Colombia, Haiti, Honduras (Fig. 5) and Nicaragua (Fig. 6). The data for these countries include both dengue and DHF from the PAHO. As the dengue pandemic did not intensify in the Americas until the 1980s, following the termination of Aedes aegypti eradication programs, these 4 countries averaged only about 14 years worth of dengue/ DHF case data. In the detrended dataset, statistically significant correlations existed for Colombia, Haiti and Nicaragua (cf. Table 2).

Table 2 illustrates differences between the PAHO and GIDEON data sets. There are only a few countries where PAHO and GIDEON data are available for the same years, and even then, the actual numbers of reported cases differ, as shown by the different correlation coefficients.

\section{DISCUSSION}

A previous study has shown that there is good agreement between the observed (www.cdc.gov/ncidod/ dvbid/dengue/map-distribution-2000.htm) and the modelled global distribution of Aedes aegypti. Seasonal fluctuations in mosquito abundance also compare well with observed data (Hopp \& Foley 2001). This analysis shows that in several countries there is a strong relationship between climate-induced varia- 


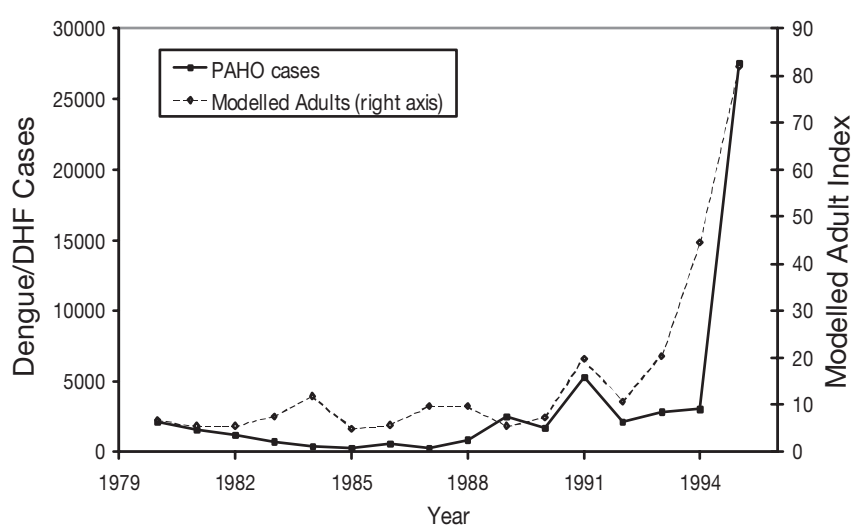

Fig. 5. Honduras: Dengue/DHF cases (PAHO dataset) and modelled adult mosquito (Aedes aegypti) index. Correlation coefficient $=0.91^{*}\left(0.88^{*} \text { detrended }\right)_{i}{ }^{*} \mathrm{p}<0.05$

tions in modelled mosquito densities and dengue/DHF cases (Figs. 3 to 6). On average, Asian countries have more years of dengue/DHF case data than American countries, and those nations with more years of data tend to have more significant results (Tables 1 \& 2). Following the Southeast Asian dengue pandemic at the conclusion of World War II, dengue/DHF has become an important infectious disease in Asia, and it is the leading cause of childhood mortality in many countries. Only in the last few decades has dengue reemerged as a health threat in the Americas, following the termination of Ae. aegypti eradication efforts begun in the 1950s and 1960s (Gubler \& Clark 1995, WHO 1998).

In several of the larger countries analyzed (Australia, Brazil, China and the United States), the correlations between the modelled mosquito densities and reported dengue/DHF caseloads are not significant. This

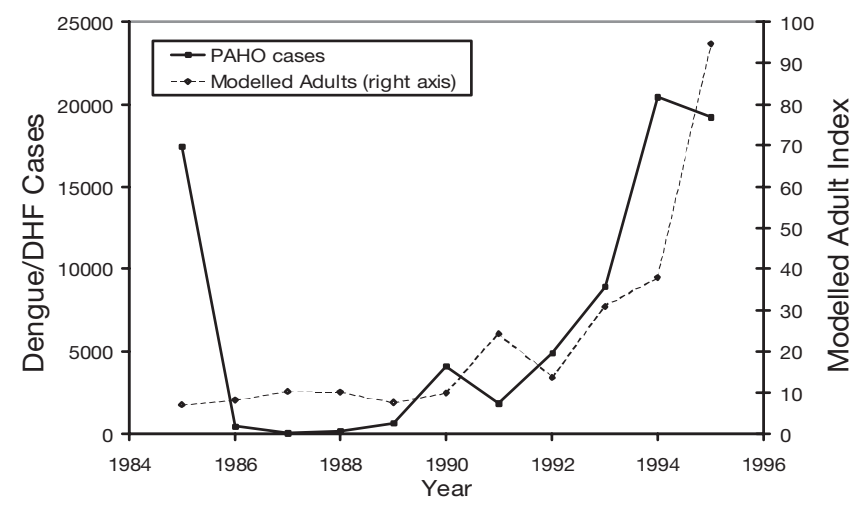

Fig. 6. Nicaragua: Dengue/DHF cases (PAHO dataset) and modelled adult mosquito (Aedes aegypti) index. Correlation coefficient $=0.64{ }^{*}(0.49$ detrended $){ }^{*} p<0.05$

is likely due to the fact that dengue cases only occur in limited regions of these countries (e.g. SE ChinaGuangdong Province and Hainan Island), but were compared to the modelled mosquito data averaged over the entire country. A similar problem occurs in the United States, where most of the cases occur in the SE and many are imported cases, a factor not considered in this study. Further studies could use sub-country data, such as state or provincial dengue reports. Working at finer spatial resolutions in the mosquito model would allow the inclusion of smaller countries and islands where dengue is endemic.

In analyzing the relationships between the modelled mosquito densities and the reported dengue/DHF caseloads, factors such as increases in human population over the years were removed by detrending the caseload data. Detrending the modelled mosquito densities eliminated factors such as general warming

Table 1. Correlation coefficients (CC) and detrended CC between modelled adult mosquito (Aedes aegypti) densities and Asian dengue/DHF cases from annual GIDEON data. ${ }^{*} p<0.05$

\begin{tabular}{|c|c|c|c|c|c|}
\hline & $\mathrm{CC}$ & Detrended CC & Years of data & Dengue & DHF \\
\hline Australia & 0.30 & 0.093 & 5 (1991-95) & $\mathrm{x}$ & \\
\hline Cambodia & $0.54^{*}$ & 0.42 & $16(1980-95)$ & & $\mathrm{x}$ \\
\hline China & -0.62 & -0.82 & $9(1986,1988-95)$ & & $\mathrm{x}$ \\
\hline India & 0.29 & 0.55 & $7(1976,1982,1991-95)$ & $\mathrm{x}$ & $\mathrm{x}$ \\
\hline Indonesia & $0.77^{*}$ & $0.58^{*}$ & $27(1968-95)$ & & $\mathrm{x}$ \\
\hline Laos & $0.54^{*}$ & 0.50 & $14(1981,1983-95)$ & & $\mathrm{x}$ \\
\hline Malaysia $^{a}$ & $0.82^{*}$ & 0.14 & $11(1968,1970,1973-80,1995)$ & $\mathrm{x}$ & \\
\hline Malaysia $^{\text {b }}$ & $0.57^{*}$ & 0.19 & $26(1968,1970,1973-95)$ & $\mathrm{x}$ & $\mathrm{x}$ \\
\hline Myanmar & 0.18 & -0.032 & $26(1970-95)$ & & $\mathrm{x}$ \\
\hline Philippines & $0.36^{*}$ & 0.21 & $37(1958-95)$ & & $\mathrm{x}$ \\
\hline Sri Lanka & -0.17 & -0.38 & $15(1965-70,1977,1988-95)$ & & $\mathrm{x}$ \\
\hline Thailand & $0.68^{*}$ & $0.43^{*}$ & $37(1959-95)$ & & $\mathrm{x}$ \\
\hline Vietnam & $0.43^{*}$ & $0.51^{*}$ & $21(1975-95)$ & & $\mathrm{x}$ \\
\hline
\end{tabular}


Table 2. Correlation coefficients (CC) and detrended CC between modelled adult mosquito (Aedes aegypti) densities and annual dengue/DHF data for the Americas from the GIDEON and PAHO datasets. ${ }^{*} \mathrm{p}<0.05$

\begin{tabular}{|c|c|c|c|c|c|c|}
\hline & $\mathrm{CC}$ & $\begin{array}{c}\text { GIDEON } \\
\text { Detrended CC }\end{array}$ & Years of data & $\mathrm{CC}$ & $\begin{array}{c}\text { PAHO } \\
\text { Detrended CC }\end{array}$ & Years of data \\
\hline Belize & -0.13 & 0.19 & $\begin{array}{l}8(1978,1980-84,1986, \\
1988-95)\end{array}$ & 0.013 & 0.12 & $\begin{array}{l}8(1980-84,1986, \\
1990,1995)\end{array}$ \\
\hline Bolivia & -0.24 & -0.027 & $8(1987-94)$ & & & \\
\hline Brazil & 0.55 & 0.48 & $11(1982,1986-95)$ & 0.55 & 0.48 & $11(1982,1986-95)$ \\
\hline Colombia & 0.17 & 0.075 & $16(1980-95)$ & $0.66^{*}$ & $0.65^{*}$ & $16(1980-95)$ \\
\hline Cuba & -0.55 & -0.51 & $5(1977-81)$ & & & \\
\hline Dominican Republic & 0.29 & 0.29 & $\begin{array}{l}25(1960-65,1969,1971 \\
1978,1980-95)\end{array}$ & 0.38 & 0.37 & $16(1980-95)$ \\
\hline Ecuador & 0.35 & -0.73 & $8(1988-95)$ & 0.58 & -0.61 & $8(1988-95)$ \\
\hline El Salvador & 0.19 & -0.21 & $18(1978-95)$ & -0.16 & 0.34 & $16(1980-95)$ \\
\hline French Guiana & 0.35 & 0.032 & $4(1986,1991-92,1995)$ & 0.52 & 0.49 & $7(1986,1990-95)$ \\
\hline Guatemala & 0.28 & -0.21 & $\begin{array}{l}15(1974,1978,1980-83 \\
1987-95)\end{array}$ & 0.21 & -0.23 & $\begin{array}{l}13(1980-83 \\
1987-95)\end{array}$ \\
\hline Haiti & 0.12 & 0.12 & $\begin{array}{l}12(1972-75,1977-78 \\
1980-85,1988-92,1994)\end{array}$ & $0.58^{*}$ & $0.59^{*}$ & $\begin{array}{l}12(1980-85 \\
1990-95)\end{array}$ \\
\hline Honduras & 0.10 & 0.37 & $18(1978-95)$ & $0.91^{*}$ & $0.88^{*}$ & $16(1980-95)$ \\
\hline Mexico & -0.13 & 0.080 & $\begin{array}{l}16(1971,1980-92, \\
1994-95)\end{array}$ & -0.37 & 0.34 & $16(1980-95)$ \\
\hline Nicaragua & $0.64^{*}$ & 0.49 & $11(1985-95)$ & $0.64^{*}$ & 0.49 & 11 (1985-95) \\
\hline Paraguay & -0.46 & -0.48 & $8(1988-95)$ & & & \\
\hline Peru & -0.22 & -0.31 & $8(1988-95)$ & -0.68 & -0.49 & $6(1990-95)$ \\
\hline Surinam & 0.62 & 0.51 & $10(1978,1980,1988-95)$ & 0.17 & 0.052 & $12(1981-82,1986-95)$ \\
\hline Trinidad and Tobago & 0.26 & 0.0027 & $17(1978,1980-95)$ & 0.44 & 0.27 & $15(1981-95)$ \\
\hline USA & & & & 0.12 & 0.12 & $15(1980-90,1992-95)$ \\
\hline Venezuela & 0.073 & 0.048 & $\begin{array}{l}26(1964-73,1978 \\
1980-85,1987-95)\end{array}$ & 0.16 & 0.18 & $15(1980-85,1987-95)$ \\
\hline
\end{tabular}

trends in the climate, since rising temperatures may accelerate the mosquito's rates of development and, consequently, one might expect increases in mosquito abundances. This is possibly the case in Thailand and Indonesia (Figs. 3 \& 4) with a rising trend in modelled mosquito densities over the last several decades. Detrending the data and removing this temperature effect, however, resulted in fewer statistically significant correlations coefficients between mosquitoes and case data in the Asian and American countries (Tables 1 \& 2). As there are fewer years of dengue/ DHF data in the Americas compared to Asia, the temperature trend would not be expected to be as significant as it is in the Asian countries. Therefore, it is not unexpected that the correlation coefficients of fewer American countries are affected by the detrended data as compared to the Asian countries.

The issue of health data reliability is illustrated by a comparison of the PAHO and GIDEON data for countries in the Americas (Table 2). The 2 data sets often include different years of coverage for a particular country, and even when the same years are covered, the dengue caseload data may differ. This results in differ- ent correlation coefficients for the PAHO and GIDEON data for the same country. All of the statistically significant correlation coefficients with the modelled mosquito densities in the Americas were observed with the PAHO data. Use of DHF data exclusively, where available, may also be more reliable, as all of the statistically significant correlations in Asian countries were based on DHF data. Further studies using higher resolution dengue/DHF case data, both spatially (subcountry data) and temporally (monthly, as opposed to the annual data used in this study) may further elucidate relationships between the climate-driven modelled mosquito densities and dengue case data.

The significant relationships between modelled mosquito densities and dengue/DHF case data in several countries are illustrated by the correlation of the yearto-year fluctuations in the modelled densities with the peaks in the reported dengue/DHF cases (Figs. 3 to 6). In Thailand (Fig. 3) and Indonesia (Fig. 4) there are significant increases in the DHF cases reported over the decades. This is likely due to increases in the human population, circulation of additional dengue viruses, and increases in climate-dependent mosquito densi- 
ties. The fluctuations in the modelled mosquito densities in Figs. 3 to 6 are entirely due to climatic variations, as we have excluded other factors that might affect mosquito abundances.

In this study, the mosquito model was driven with historical climate data. But long-lead seasonal climate forecasts (such as those conducted to predict El Niño and La Niña events) could also be used to forecast changes in mosquito densities. Results from this analysis suggest that such forecasts could, in turn, be used to anticipate dengue caseloads.

As no vaccine exists yet, the primary means of controlling dengue is by controlling the mosquitoes (WHO 1998). Using seasonal climate forecasts to drive the mosquito model, mosquito densities relative to historical densities may be predicted several months in advance. For example, a mosquito forecast (posted on a web site) for higher-than-normal densities in a particular region, could motivate health officials to alert the public to the increased risk of acquiring dengue, and increase mosquito control efforts. A mosquito density forecast for a particular area, together with information on which dengue viruses are circulating, the human population's immunity, as well as knowledge of current mosquito control efforts, can be a component of an early warning system for dengue. This potential dengue mosquito forecasting tool may thus help prevent dengue outbreaks.

Acknowledgements. We thank our colleagues at the University of Wisconsin-Madison and the International Research Institute for Climate Prediction (IRI) for their very helpful suggestions for this manuscript. We would also like to thank the CPEPers (Climate, People and Environmental Program) for their assistance and advice throughout this research. This work was supported by the Institute for Environmental Studies at the University of Wisconsin-Madison through the Laurel Foundation.

\section{LITERATURE CITED}

Berger SA (1995) Computer program for diagnosing and teaching geographic medicine. J Travel Med 2:199-203

Christophers SR (1960) Aedes aegypti (L.) The yellow fever mosquito. Its life history, bionomics and structure. Cambridge University Press, Cambridge

Focks DA, Haile DG, Daniels E, Mount GA (1993) Dynamic life table model for Aedes aegypti (Diptera: Culicidae): analysis of the literature and model development. J Med Entomol 30:1003-1017

Focks DA, Haile DG, Daniels E, Mount GA (1993) Dynamic life table model for Aedes aegypti (Diptera: Culicidae): simulation results and validation. J Med Entomol 30: 1018-1028

Gagnon AS, Bush ABG, Smoyer-Tomic KE (2001) Dengue epidemics and the El Niño Southern Oscillation. Clim Res 19:35-43

Gubler DJ (1997) Dengue and dengue hemorrhagic fever: its history and resurgence as a global public health problem.
In: Gubler DJ, Kuno G (eds) Dengue and dengue hemorrhagic fever, CAB International, New York

Gubler DJ (1998) Resurgent vector-borne diseases as a global health problem. Emerg Infect Dis 4:442-450

Gubler DJ, Clark GG (1995) Dengue/dengue hemorrhagic fever: the emergence of a global health problem. Emerg Infect Dis 1:55-57

Hales S, Weinstein P, Souares Y, Woodward A (1999) El Niño and the dynamics of vectorborne disease transmission. Environ Health Perspect 107:99-102

Hopp MJ, Foley JA (2001) Global-scale relationships between climate and the dengue fever vector, Aedes aegypti. Clim Change 48:441-463

Jetten TH, Focks DA (1997) Potential changes in the distribution of dengue transmission under climate warming. Amer J Trop Med Hyg 57:285-297.

Martens WJM, Jetten TH, Focks DA (1997) Sensitivity of malaria, schistosomiasis and dengue to global warming. Clim Change 35:145-156.

New MG, Hulme M, Jones PD (1999) Representing 20th century space-time climate variability. I: Development of a 1961-1990 mean monthly terrestrial climatology. J Clim $12: 829-856$

New MG, Hulme M, Jones PD (2000) Representing 20th century space-time climate variability. II: Development of 1901-1996 monthly terrestrial climate fields. J Clim 13: $2217-2238$

PAHO (1994) Dengue and dengue hemorrhagic fever in the Americas: Guidelines for prevention and control. Pan American Health Organization Sci. Pub. No. 548, Washington, DC

PAHO (1997) Dengue and dengue hemorrhagic fever (DHF) cases reported in the Americas, 1990-1996. Pan American Health Organization, Communicable Diseases Program, Washington, DC

Patz JA, Martens WJM, Focks DA, Jetten TH (1998) Dengue fever epidemic potential as projected by general circulation models of global climate change. Environ Health Perspect 106:147-153.

Pinheiro FP, Chuit R (1998) Emergence of dengue hemorrhagic fever in the Americas. Infect in Med 15:244-251

Prentice IC, Sykes MT, Cramer W (1993) A simulation model for the transient effects of climate change on forest landscapes. Ecol Model 65:51-70

Reiter P (1998) Global-warming and vector-borne disease in temperate regions and at high altitude. Lancet 351: 839-840

Reiter P (2001) Climate change and mosquito-borne disease. Environ Health Perspect 109 (Suppl 1):141-161

Reiter P, Lathrop S, Bunning M, Biggerstaff B and 16 others (2003) Texas lifestyle limits transmission of dengue virus. Emerg Infect Dis 9:86-89

Rigau-Pérez JG, Clark GG, Gubler DJ, Reiter P, Sanders EJ, Vorndam AV (1998) Dengue and dengue haemorrhagic fever. Lancet 352:971-977

Rodhain F, Rosen L (1997) Mosquito vectors and dengue virus-vector relationships. In: Gubler DJ, Kuno G (eds) Dengue and dengue hemorrhagic fever, CAB International, New York

Rueda LM, Patel KJ, Axtell RC, Stinner RE (1990) Temperature-dependent development and survival rates of Culex quinquefasciatus and Aedes aegypti (Diptera: Culicidae). J Med Entomol 27:892-898

Sharpe PJH, DeMichele DW (1977) Reaction kinetics of poikilotherm development. J Theor Biol 64:649-670

Shope R (1991) Global climate change and infectious diseases. Environ Health Perspect 96:171-174 
Watts DM, Burke DS, Harrison BA, Whitmore RE, Nisalak A (1987) Effect of temperature on the vector efficiency of Aedes aegypti for Dengue 2 virus. Am J Trop Med Hyg 36: $143-152$

WHO (1998) Dengue and dengue haemorrhagic fever. WHO Fact Sheet No. 117. Available from: URL: www.who.int/ inf-fs/en/fact117.html

Editorial responsibility: Andrew Comrie,

Tucson, Arizona, USA
WHO (1999) Guidelines for the treatment of dengue fever/ dengue haemorrhagic fever in small hospitals. WHO Regional Office SE Asia, New Delhi

WHO (2000) Dengue/dengue haemorrhagic fever. Wkly Epidemiol Rec 75:193-200

WHO (2001) Chiangmai declaration on dengue/dengue haemorrhagic fever. Wkly Epidemiol Rec 4:29-30

Submitted: September 8, 2001; Accepted: July 15, 2003

Proofs received from author(s): September 11, 2003 